\title{
27. GEOCHEMISTRY AND RARE-EARTH ELEMENT ABUNDANCES OF BASALTS FROM SITES 482, 483, AND 485 IN THE GULF OF CALIFORNIA ${ }^{1}$
}

\author{
B. P. Zolotarev and E. M. Margolin, Geological Institute of the USSR Academy of Sciences, Moscow, USSR
}

\section{INTRODUCTION}

Petrological investigations of oceanic rocks carried out during the past several years have shed considerable light on the geochemistry of basalts from various structural provinces in the ocean basins. It is evident from these studies that variations in chemistry exist between volcanics in different provinces and even within individual provinces.

The midoceanic ridges, for example, consist mostly of slightly differentiated abyssal oceanic tholeiites formed during underwater fissure eruptions. The ridges abound, as a rule, in aphyric pillow lavas, but olivine or olivineplagioclase phyric varieties occur less frequently. In addition, aphyric to porphyritic massive basalts are common in most crustal sections (Hall and Robinson, 1979) and even predominate along some portions of the ridge. All of these rocks are characterized by a low alkaline element content, and many show a low content of titanium and a relatively high magnesium content. A high $\mathrm{Na}_{2} \mathrm{O} / \mathrm{K}_{2} \mathrm{O}$ ratio, usually reaching 20 and sometimes even 50 , is peculiar to them.

Despite the fact that the rocks composing the midoceanic ridges belong to one petrochemical type (abyssal oceanic tholeiites), it has been observed that basalts found along the midoceanic ridges display subtle variations of contents of magnesium, iron, titanium, and alkaline elements. These variations appear to be results of lateral heterogeneity and (or) of different degrees of partial melting in the upper mantle. When judged on the basis of magmatic characteristics, it is apparent that primary magmas formed under sections of the midocean ridges with similar structures are generated at about the same depths. However, there are areas with abnormally thick crust. On the Mid-Atlantic Ridge these are the Azores and Iceland, and Easter Island on the East Pacific Rise. Volcanic rocks in such areas formed not only by fissure eruptions, but by central eruptions as well. They differ from typical abyssal oceanic tholeiites in displaying greater differentiation (to dacites) and higher concentrations of iron, titanium, and alkaline elements. The locus of magma formation in such areas is assumed to be deeper than under parts of the ridges characterized by more typical structures (Zolotarev, 1979).

Basalts of the East Pacific Rise appear to be more evolved than those on the Mid-Atlantic Ridge. Correla-

\footnotetext{
${ }^{1}$ Lewis, B. T. R., Robinson, P., et al., Init. Repts. DSDP, 65: Washington (U.S. Govt. Printing Office).
}

tion of chemistry of volcanics of the Mid-Atlantic Ridge and East Pacific Rise reveals certain petrochemical differences between the two provinces: basalts of the Pacific Ocean are characterized by somewhat higher iron and titanium contents than are those of the Atlantic Ocean. However, rather considerable variations in the abundance of these elements are apparent in both provinces. In cases where the iron content of rocks increases, the magnesium content decreases and titanium concentration grows higher.

It follows from the concept of sea-floor spreading that the oceanic crust is formed along the midoceanic ridges. It is thus important to determine both the structure of the ridge and the magmatic evolution of the rocks of which it is composed as a function of position along its length.

The region under investigation in the present study is situated at the northern end of the East Pacific Rise on young crust at the mouth of the Gulf of California. Our objective is to determine the major element and rareearth element geochemistry of the basalts composing the upper part of Layer 2 in this region for comparison with basalts from other sections of the ridge. The samples examined were selected during Deep Sea Drilling Project (DSDP) Leg 65 from the least altered basalts recovered from Sites 482, 483, and 485 .

\section{METHODS}

Several methods were used in this study to determine basalt composition. In addition to the thin section and XRF studies conducted on shipboard during Leg 65 (see site summaries chapters, this volume), some analyses were done at the Analytical Chemistry Laboratory of the Geological Institute of the USSR Academy of Sciences, using the standard wet-chemical techniques described by Zolotarev et al. (1979).

Rare-earth element contents were measured by instrumental neutron-activation analysis (INAA) in which 20 -mg samples were bombarded for 24 hours by thermal neutrons in a nuclear reactor. The gamma-ray activity of the samples was then measured by means of a gamma-ray spectrometer with $\mathrm{Ge}(\mathrm{Li})$ semiconductor detectors 7 to 10 $(\mathrm{La}, \mathrm{Sm}, \mathrm{Yb}, \mathrm{Lu})$ and $20(\mathrm{C}, \mathrm{Eu}, \mathrm{Tb})$ days after irradiation. A more detailed description of technique of preparing the specimens, irradiation, estimation, and treatment of data has been done previously (Zaitsev et al., 1978). Calibration of the results of the instrumental neutron-activation analysis was carried out through analysis of some standards of the Soviet Union and National Geochemical Center of France (CT-1, CTD-1A, DRN, and CA). Basaltic Br from the National Geochemical Center of France was used as a petrologic standard. Reproducibility of the obtained results is characterized by the following standard deviations: $\mathrm{La}-0.23, \mathrm{Ce}-0.87, \mathrm{Sm}-0.26, \mathrm{Eu}-0.13$, $\mathrm{Tb}-0.11, \mathrm{Yb}-0.45, \mathrm{Lu}-0.09 \mathrm{ppm}$. Comparison of obtained data of the instrumental neutron-activation analysis with values for $\mathrm{Br}$ showed an absence of appreciable systematic error, for a confidence limit of 0.95 . 


\section{RESULTS AND DISCUSSION}

The region of study of DSDP Leg 65 is in the mouth of the Gulf of California. Four sites were chosen for drilling. One of them is situated on the western slope of the East Pacific Rise (Site 483), and two are on the eastern slope (Sites 482,485 ). In addition, an attempt was made to drill a "magnetic diapir" in the northern trough of the Tamayo Fracture Zone (Site 484). Altogether 15 holes were drilled, but only 9 of them penetrated the basement. The greatest basement penetration was in Holes 483B, 482B, and 485A. Although massive basalts generally predominate, pillow basalts alternating with flows of massive basalt were encountered in many of the holes drilled, and pillow basalts predominate over massive basalts in several of the holes drilled at Site 483.

Aphyric and porphyritic varieties can be observed among both types of basalts. Volumetrically, aphyric basalts are slightly predominant. Petrographically and texturally, the basalts penetrated at Sites 482,483 , and 485 are rather similar. Pillow basalts contain, as a rule, a small amount of microphenocrysts of plagioclase and olivine, sometimes forming glomerophyric clusters. The amount of plagioclase microphenocrysts is 1 to $3 \%$, olivine, up to $1 \%$ by volume. Their dimensions are 0.02 to $0.7 \mathrm{~mm}$ and in rare cases over $1 \mathrm{~mm}$. Sometimes olivine is replaced by secondary minerals (smectite, celadonite, and more rarely, carbonate). The opaque mineral content varies from 2 to $5 \%$. Most frequently, it is represented by specks or small xenomorphic patches in the groundmass. The groundmasses of typical rocks are represented by laths of plagioclase and clinopyroxene, the interstices between them being filled with volcanic glass. The porosity varies from 1 to $5-7 \%$. Pores are frequently filled with smectite. Sometimes the pores are lined with smectite and filled with calcite.

The groundmass textures of the pillow basalts are diverse, but most frequently are intergranular or interstitial. The glassy intervals, on the other hand, tend to be subvariolitic, variolitic, or hyalopilitic.

Massive basalts are, as a rule, well crystallized. They incorporate both the aphyric and the sparsely porphyritic varieties. Phenocrysts are represented by plagioclase $(1-5 \%)$, less frequently by olivine (up to $1 \%$ ). The size of the phenocrysts does not exceed $1 \mathrm{~mm}$. The groundmass is composed of plagioclase laths $(30-50 \%)$, clinopyroxene $(30-40 \%)$, opaques $(3-7 \%)$, and sometimes volcanic glass filling the interstices between plagioclase and clinopyroxene.

Secondary minerals are represented by smectite and carbonate, which, as a rule, partly replace volcanic interstitial glass. The texture of massive basalts is intergranular, interstitial, subophitic or ophitic, and sometimes glomeroporphyritic.

In Holes 482B and 485A eight independent lithologic units were distinguished among basalts, and in Hole 483B, nine. The chemical composition of basalts composing these units varies within insignificant limits, and no significant change in chemistry was observed with depth. Unit 4 in all three holes, however, is characterized by somewhat lesser contents of silica, titanium, and ferrous iron and by higher contents of alumina, magnesium, and $\mathrm{H}_{2} \mathrm{O}^{+}$, even though the phenocryst contents are quite variable (Unit 4 is aphyric in Hole 483B and sparsely plagioclase or plagioclase-olivine phyric in Holes $482 \mathrm{~B}$ and $485 \mathrm{~A}$ ). This pattern is especially pronounced in the massive aphyric basalts of Hole 482B. A slight trend toward decreasing silica and calcium and an increase in titanium was also observed with depth in this hole (Fig. 1). It should be emphasized, however, that the established variations in composition of the basalts composing the various units in these holes lie within limits typical of oceanic abyssal tholeiites.

The basalt compositions determined in this study are presented in Table 1, and both two- and three-component diagrams based on the major oxide data are shown in Figures 2 through 8. The three-component AFM diagram (Fig. 2) shows that the basalts analyzed are tholeiitic with a poorly developed differentiation trend. The ratios of the some of the rock-forming elements ( $\mathrm{FeO}$ : $\mathrm{MgO}, \mathrm{Al}_{2} \mathrm{O}_{3}: \mathrm{TiO}_{2}, \mathrm{Al}_{2} \mathrm{O}_{3}: \mathrm{MgO}, \mathrm{Al}_{2} \mathrm{O}_{3}: \mathrm{CaO}, \mathrm{MgO}: \mathrm{CaO}$, $\mathrm{K}_{2} \mathrm{O}: \mathrm{TiO}_{2}$ ) also show that the basalts are abyssal tholeiites. As can be seen in Figures 3 and 4, the pairs $\mathrm{FeO}-$ $\mathrm{MgO}$ and $\mathrm{Al}_{2} \mathrm{O}_{3}-\mathrm{TiO}_{2}$ show a weak inverse correlation while the pairs $\mathrm{Al}_{2} \mathrm{O}_{3}-\mathrm{MgO}, \mathrm{Al}_{2} \mathrm{O}_{3}-\mathrm{CaO}, \mathrm{MgO}-\mathrm{CaO}$, and $\mathrm{K}_{2} \mathrm{O}-\mathrm{TiO}_{2}$ show practically no correlation whatsoever (Figs. 5-8). However, one trend is clearly recognized in all these diagrams. Although the chemical composition of basalts at Sites 483 and 485 are similar to each other, they differ from that at Site 482 . On the whole, basalts of Site 482 contain greater amounts of $\mathrm{MgO}, \mathrm{Al}_{2} \mathrm{O}_{3}$, and $\mathrm{CaO}$ and somewhat lower concentrations of $\mathrm{FeO}$ and $\mathrm{TiO}_{2}$. In the Miyashiro diagram (Fig. 9), practically all the analyzed basalts lie in the abyssal oceanic tholeiites field below the boundary line V separating fresh rocks from rocks altered by secondary processes. Thus, the aforementioned difference in the chemical composition of the basalts represents a difference in magma composition and can by no means be explained by alteration, particularly since only fresh samples were selected for chemical analyses.

Figure 10 reveals a slight negative correlation between parameter $t=\mathrm{Al}_{2} \mathrm{O}_{3}-\mathrm{Na}_{2} \mathrm{O} / \mathrm{TiO}_{2}$ and $\mathrm{TiO}_{2}$ content, which is characteristic of abyssal oceanic tholeiites as well. However, variation in the parameter $t$ is much smaller in this case than is characteristic of abyssal tholeiites of the Atlantic Ocean. This testifies to the lower alumina contents and to the lower degree of differentiation of basalts of the East Pacific Rise, as compared with basalts of the Mid-Atlantic Ridge.

In the a-s Dmitriev diagram (Fig. 11), the compositions of Leg 65 basalts lie in the field of abyssal oceanic tholeiites, covering its small left part. Leg 65 basalts (Fig. 11B) are enriched in mafic components, particularly in iron, titanium, and magnesium as compared to basalts of the Atlantic Ocean. We see variations in distribution of these elements in both of the compared provinces. In cases where the iron content of the rocks increases sharply, the magnesium content decreases, and titanium concentration increases. These geochemical trends may result from different degrees of differentiation of the upper mantle under the Atlantic and Pacific 


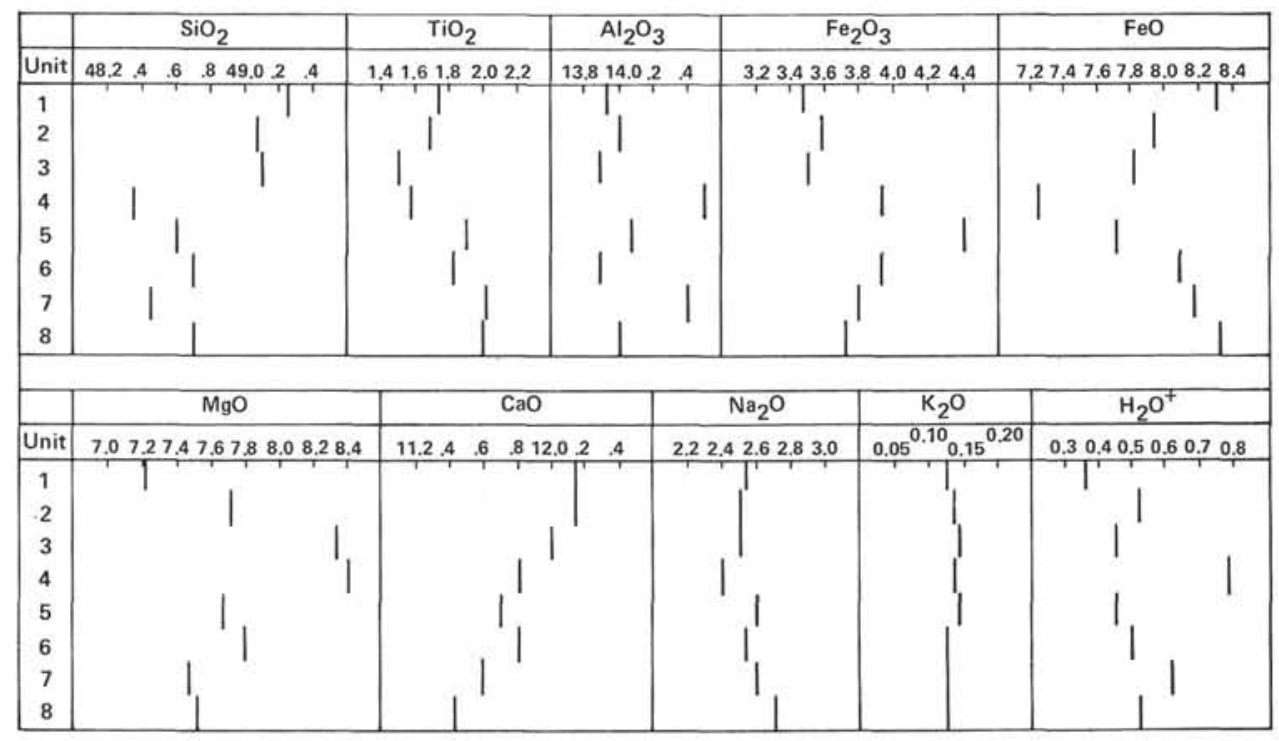

Figure 1. Major element composition of lithologic units in Hole 482B. (Values shown in wt.\%.)

oceans. The mantle substratum of the Pacific province at the levels of primary melt generation may be more differentiated and, correspondingly, more enriched in these siderophile elements than the mantle substratum of the Atlantic province, which is characterized by somewhat higher contents of lithophile elements. In Figure 11B, the compositions of basalts at Site 482 plot, on the average, in positions further from the origin than basalts at Sites 483 and 485 . When plotted in this diagram, the compositions of these basalts complement those of oceanic lherzolites (Fig. 11, Fields I, II). The conclusion is that the parental basaltic melts were formed by partial melting of oceanic lherzolites.

Differences among Leg 65 basalts can be explained by various degrees of partial melting of the mantle substratum. It would be difficult to attribute these differences to differential crystallization, because no significant differences are seen in compositions or amounts of phyric phenocrysts. Such phenocrysts are insignificant in abundance.

In an alkali-silica plot (Fig. 12), the Leg 65 basalts lie in the field of abyssal oceanic tholeiites close to a tholeiitic differentiation trend. In this diagram the compositions of basalts at Sites 482,483 , and 485 somewhat differ in the ratio of alkaline elements to silica. The interpretation of this diagram using the results of Green and Ringwood (1967) enable us to conclude that melting of basalts occurred at depths of about 20 to $25 \mathrm{~km}$, and that their subsequent evolution took place at low pressures. It follows that the formation of phenocrysts occurred not in the primary magma pocket (not at the protomagmatic stage), but in less abyssal intermediate chambers or during eruption, inside lava flows or oozes. Under such conditions, precipitation of olivine crystals or flotation of plagioclase is negligible.

The distribution of rare-earth elements in Leg 65 basalts is shown in Figure 13. Yu. A. Balashov (1976) noted that rare-earth elements are sensitive indicators of the processes of magmatic differentiation. During fractional crystallization, the factors determining behavior of rare-earth elements are crystallochemical differences (monotonic change in ionic radii from $\mathrm{La}$ to $\mathrm{Lu}$ ), whereas in the melting and postmagmatic stages REE behavior is controlled by their affinity for certain anions $\left(\mathrm{CO}_{3}^{2-}, \mathrm{F}^{-}\right.$, and $\left.\mathrm{PO}_{4}^{3-}\right)$ and dependence of their concentrations upon alkaline elements.

Fractionation of rare-earth elements plays a significant role in controlling their concentrations in volcanic rocks characterized by high contents of alkali elements, particularly potassium. The Leg 65 basalts are characterized by extremely low sodium and potassium contents and by insignificant variations in those contents. Therefore, the main factor controlling fractionation of rareearth elements is their crystallochemical differences.

Table 2 reports contents of rare-earth elements in Leg 65 basalts. These data show that all samples analyzed are characterized by relative enrichment of medium and heavy rare-earth elements.

However, two types of fractionation are identified. Rocks of Sites 483 and 485 are enriched with Eu and Lu relative to $\mathrm{Sm}$ and $\mathrm{Yb}$, respectively, whereas basalts of Site 482 show deficiencies in Eu and Lu compared with $\mathrm{Sm}$ and $\mathrm{Yb}$. These relationships are observed, on the whole, in all units at each site, except Units 2 and 4. Unit 2 of Sites 483 and 485 contains a surplus of Eu relative to $\mathrm{Sm}$ but a deficiency of Lu relative to $\mathrm{Yb}$. Similar ratios characterize Unit 4 of Sites 482 and 483 . These data suggest a somewhat different character of fractionation of rare-earth elements in Units 2 and 4, as compared with the others.

The sum of lanthanum, europium, ytterbium, lutecium, and samarium contents are plotted in Figure 14 as a function of unit number, i.e., the depth of occurrence in the second layer of the oceanic crust. Unit 4 at Site 483 is characterized by a deficiency of rare-earth elements. Despite sharp differences in total contents of lanthanum, samarium, europium, ytterbium, and lutecium at each site, these differences become less significant in basalts of the upper units and are practically insignificant in rocks of Unit 8. In other words, within various sites, 


\section{B. P. ZOLOTAREV, E. M. MARGOLIN}

Table 1. Composition of basalts from Sites 482,483 , and $485 .^{\text {a }}$

\begin{tabular}{|c|c|c|c|c|c|c|c|c|c|c|c|c|c|c|}
\hline \multirow{4}{*}{$\begin{array}{l}\text { Hole } \\
\text { Core } \\
\text { Section } \\
\text { Interval }(\mathrm{cm})\end{array}$} & \multicolumn{14}{|c|}{$482 \mathrm{~B}$} \\
\hline & 11 & 12 & 13 & 14 & 14 & 14 & 14 & 15 & 15 & 15 & 16 & 16 & 18 & 18 \\
\hline & 1 & 1 & 1 & 1 & 1 & 2 & 3 & 1 & 2 & 3 & 1 & 5 & 1 & 2 \\
\hline & $77-85$ & $107-113$ & $54-61$ & $12-20$ & $101-106$ & $94-101$ & $109-119$ & $94-100$ & $120-129$ & $20-30$ & $41-50$ & $39-49$ & $49-57$ & $44-51$ \\
\hline $\mathrm{SiO}_{2}$ & 49.58 & 50.03 & 49.31 & 49.54 & 48.98 & 48.94 & 49.42 & 49.44 & 50.17 & 50.10 & 50.16 & 50.40 & 50.02 & 50.20 \\
\hline $\mathrm{TiO}_{2}$ & 1.87 & 1.78 & 1.96 & 1.96 & 1.53 & 1.53 & 1.48 & 1.49 & 1.38 & 1.38 & 1.37 & 1.37 & 1.37 & 1.37 \\
\hline $\mathrm{Al}_{2} \mathrm{O}_{3}$ & 14.64 & 14.82 & 14.51 & 14.51 & 15.33 & 15.78 & 14.96 & 15.12 & 15.03 & 15.14 & 14.74 & 15.06 & 14.61 & 14.72 \\
\hline $\mathrm{Fe}_{2} \mathrm{O}_{3}$ & 2.02 & 2.84 & 2.16 & 2.36 & 2.31 & 2.43 & 2.04 & 1.90 & 1.77 & 2.07 & 2.34 & 2.07 & 2.19 & 2.38 \\
\hline $\mathrm{FeO}^{\circ}$ & 8.35 & 7.41 & 8.52 & 8.33 & 7.76 & 7.36 & 8.10 & 8.12 & 7.93 & 7.66 & 7.83 & 7.31 & 8.10 & 7.78 \\
\hline $\mathrm{MnO}$ & 0.19 & 0.17 & 0.21 & 0.18 & 0.18 & 0.17 & 0.19 & 0.19 & 0.17 & 0.17 & 0.17 & 0.17 & 0.17 & 0.19 \\
\hline $\mathrm{MgO}$ & 7.50 & 7.66 & 7.57 & 7.49 & 7.58 & 7.60 & 7.71 & 7.92 & 7.93 & 7.93 & 8.06 & 7.80 & 7.96 & 8.04 \\
\hline $\mathrm{CaO}$ & 12.12 & 11.72 & 12.23 & 11.75 & 11.98 & 11.64 & 12.16 & 11.98 & 12.22 & 12.10 & 12.15 & 12.03 & 12.14 & 12.14 \\
\hline $\mathrm{Na}_{2} \mathrm{O}$ & 2.57 & 2.70 & 2.57 & 2.43 & 2.43 & 2.43 & 2.30 & 2.30 & 2.30 & 2.30 & 2.16 & 2.30 & 2.43 & 2.30 \\
\hline $\mathrm{K}_{2} \mathrm{O}$ & 0.17 & 0.17 & 0.17 & 0.17 & 0.17 & 0.17 & 0.17 & 0.17 & 0.17 & 0.17 & 0.17 & 0.17 & 0.17 & 0.17 \\
\hline $\mathrm{H}_{2} \mathrm{O}^{+}$ & 0.64 & 0.59 & 0.56 & 0.39 & 1.03 & 1.06 & 0.82 & 0.74 & 0.70 & 0.52 & 0.55 & 0.48 & 0.42 & 0.46 \\
\hline $\mathrm{H}_{2} \mathrm{O}^{-}$ & 0.24 & 0.54 & 0.24 & 0.47 & 0.66 & 0.88 & 0.54 & 0.55 & 0.34 & 0.30 & 0.30 & 0.32 & 0.38 & 0.40 \\
\hline $\mathrm{P}_{2} \mathrm{O}_{5}$ & 0.05 & 0.10 & 0.09 & 0.10 & 0.07 & 0.05 & 0.07 & 0.04 & 0.05 & 0.08 & 0.05 & 0.03 & 0.09 & 0.07 \\
\hline $\mathrm{CO}_{2}$ & 0.20 & - & - & 0.25 & 0.15 & - & - & - & - & - & - & - & - & - \\
\hline $\mathrm{C}^{2}$ & - & - & - & - & 0.15 & - & - & - & - & - & - & - & - & - \\
\hline Total & 100.14 & 100.53 & 100.10 & 99.93 & 100.31 & 100.04 & 99.96 & 99.96 & 100.16 & 99.92 & 100.05 & 99.51 & 100.05 & 100.22 \\
\hline
\end{tabular}

a Values shown in weight percent.

Table 1. (Continued).

\begin{tabular}{|c|c|c|c|c|c|c|c|c|c|c|c|c|c|}
\hline \multirow{4}{*}{$\begin{array}{l}\text { Hole } \\
\text { Core } \\
\text { Section } \\
\text { Interval }(\mathrm{cm})\end{array}$} & \multicolumn{8}{|c|}{$482 \mathrm{D}$} & \multirow{2}{*}{$\frac{482 \mathrm{~F}}{5}$} & \multicolumn{4}{|c|}{483} \\
\hline & 8 & 9 & 9 & 10 & 11 & 12 & 12 & 13 & & 14 & 15 & 16 & 17 \\
\hline & 1 & 1 & 2 & 1 & 1 & 1 & 3 & 1 & 1 & 1 & 1 & 1 & 2 \\
\hline & $115-124$ & $59-68$ & $130-138$ & $133-139$ & $110-118$ & $80-87$ & $104-108$ & $59-70$ & $100-108$ & $13-21$ & $121-130$ & $71-83$ & $120-128$ \\
\hline $\mathrm{SiO}_{2}$ & 47.66 & 48.76 & 48.51 & 48.46 & 48.33 & 48.50 & 49.50 & 49.19 & 48.79 & 49.25 & 49.05 & 49.03 & 47.87 \\
\hline $\mathrm{TiO}_{2}$ & 1.87 & 1.86 & 1.78 & 1.86 & 1.44 & 1.62 & 1.44 & 1.53 & 2.04 & 1.33 & 1.44 & 1.43 & 1.10 \\
\hline $\mathrm{Al}_{2} \mathrm{O}_{3}$ & 11.56 & 12.85 & 12.73 & 12.38 & 13.48 & 13.82 & 13.86 & 13.59 & 13.02 & 13.22 & 13.56 & 12.49 & 15.42 \\
\hline $\mathrm{Fe}_{2} \mathrm{O}_{3}$ & 7.00 & 4.87 & 4.61 & 5.22 & 4.24 & 3.95 & 3.69 & 4.30 & 4.71 & 5.15 & 4.30 & 4.53 & 3.17 \\
\hline $\mathrm{FeO}$ & 7.18 & 7.80 & 7.68 & 7.59 & 8.00 & 7.74 & 7.92 & 7.12 & 7.35 & 7.00 & 6.91 & 8.01 & 6.75 \\
\hline $\mathrm{MnO}$ & 0.17 & 0.17 & 0.20 & 0.17 & 0.15 & 0.14 & 0.15 & 0.14 & 0.14 & 0.15 & 0.14 & 0.15 & 0.13 \\
\hline $\mathrm{MgO}$ & 7.49 & 7.81 & 7.54 & 8.15 & 8.73 & 8.36 & 7.99 & 8.27 & 7.78 & 7.60 & 8.14 & 9.11 & 9.98 \\
\hline $\mathrm{CaO}$ & 12.49 & 11.89 & 12.34 & 12.01 & 12.10 & 11.83 & 12.35 & 11.81 & 11.60 & 12.71 & 12.79 & 11.83 & 12.34 \\
\hline $\mathrm{Na}_{2} \mathrm{O}$ & 2.43 & 2.57 & 2.57 & 2.57 & 2.41 & 2.57 & 2.43 & 2.57 & 2.85 & 2.57 & 2.70 & 2.57 & 2.30 \\
\hline $\mathrm{K}_{2} \mathrm{O}$ & 0.13 & 0.17 & 0.13 & 0.13 & 0.10 & 0.13 & 0.13 & 0.13 & 0.17 & 0.17 & 0.13 & 0.13 & 0.09 \\
\hline $\mathrm{H}_{2} \mathrm{O}^{+}$ & 0.28 & 0.55 & 0.74 & 0.61 & 0.43 & 0.72 & 0.07 & 0.51 & 0.51 & 0.37 & 0.36 & 0.38 & 0.65 \\
\hline $\mathrm{H}_{2} \mathrm{O}^{-}$ & 1.35 & 1.09 & 1.07 & 1.06 & 0.90 & 0.95 & 0.82 & 1.20 & 0.86 & 0.82 & 0.79 & 0.68 & 0.57 \\
\hline $\mathrm{P}_{2} \mathrm{O}_{5}$ & 0.14 & 0.01 & 0.01 & 0.05 & 0.03 & 0.01 & 0.08 & 0.01 & 0.15 & 0.02 & 0.01 & 0.01 & 0.01 \\
\hline C & - & - & - & - & - & - & - & - & - & - & - & - & - \\
\hline Total & 99.75 & 100.40 & 100.26 & 100.26 & 100.34 & 100.34 & 100.43 & 100.37 & 100.17 & 100.36 & 100.32 & 100.35 & 100.38 \\
\hline
\end{tabular}

Table 1. (Continued).

\begin{tabular}{|c|c|c|c|c|c|c|c|c|c|c|c|c|c|c|}
\hline \multirow{4}{*}{$\begin{array}{l}\text { Hole } \\
\text { Core } \\
\text { Section } \\
\text { Interval }(\mathrm{cm})\end{array}$} & \multicolumn{5}{|c|}{ 483B } & \multirow{2}{*}{$\frac{483 C}{4}$} & \multicolumn{8}{|c|}{$485 \mathrm{~A}$} \\
\hline & 27 & 27 & 30 & 32 & 32 & & 11 & 11 & 12 & 13 & 14 & 18 & 23 & 23 \\
\hline & 2 & 3 & 1 & 1 & 3 & 3 & 1 & 3 & 1 & 1 & 1 & 1 & 1 & 2 \\
\hline & $10-21$ & $15-25$ & $70-78$ & $110-119$ & $33-40$ & $3-14$ & $13-26$ & $140-148$ & $21-23$ & $35-42$ & $43-56$ & $9-20$ & $66-76$ & $38-50$ \\
\hline $\mathrm{SiO}_{2}$ & 48.66 & 49.02 & 49.13 & 48.93 & 48.52 & 49.62 & 46.78 & 48.78 & 49.36 & 48.87 & 49.04 & 48.64 & 46.67 & 48.43 \\
\hline $\mathrm{TiO}_{2}$ & 2.14 & 1.87 & 1.79 & 1.70 & 1.61 & 1.45 & 2.21 & 1.95 & 2.08 & 1.91 & 1.95 & 2.16 & 2.38 & 2.17 \\
\hline $\mathrm{Al}_{2} \mathrm{O}_{3}$ & 13.72 & 14.07 & 14.38 & 15.08 & 14.69 & 14.00 & 14.13 & 12.85 & 13.53 & 13.85 & 13.70 & 13.87 & 12.31 & 13.69 \\
\hline $\mathrm{Fe}_{2} \mathrm{O}_{3}$ & 4.64 & 3.56 & 3.77 & 4.69 & 4.96 & 3.41 & 5.68 & 4.23 & 3.55 & 2.87 & 3.55 & 3.62 & 5.26 & 4.60 \\
\hline $\mathrm{FeO}$ & 8.48 & 8.48 & 7.66 & 5.90 & 5.76 & 7.84 & 6.88 & 9.68 & 9.08 & 9.23 & 9.06 & 8.98 & 9.25 & 8.29 \\
\hline $\mathrm{MnO}$ & 0.18 & 0.18 & 0.21 & 0.19 & 0.18 & 0.17 & 0.11 & 0.22 & 0.18 & 0.22 & 0.20 & 0.17 & 0.15 & 0.20 \\
\hline $\mathrm{MgO}$ & 7.53 & 7.06 & 7.90 & 7.47 & 7.65 & 7.66 & 7.42 & 6.85 & 6.84 & 6.94 & 7.12 & 7.03 & 7.44 & 7.30 \\
\hline $\mathrm{CaO}$ & 11.02 & 11.87 & 11.97 & 11.96 & 12.09 & 12.46 & 9.71 & 12.20 & 11.67 & 12.56 & 11.71 & 11.69 & 11.03 & 11.37 \\
\hline $\mathrm{Na}_{2} \mathrm{O}$ & 2.82 & 2.82 & 2.70 & 2.82 & 2.70 & 2.60 & 2.94 & 2.49 & 2.70 & 2.51 & 2.61 & 2.61 & 2.70 & 2.61 \\
\hline $\mathrm{K}_{2} \mathrm{O}$ & 0.15 & 0.15 & 0.15 & 0.31 & 0.20 & 0.10 & 0.12 & 0.15 & 0.12 & 0.12 & 0.06 & 0.12 & 0.20 & 0.12 \\
\hline $\mathrm{H}_{2} \mathrm{O}^{+}$ & 0.56 & 0.72 & 0.39 & 0.52 & 0.67 & 0.18 & 1.55 & 0.35 & 0.33 & 0.41 & 0.29 & 0.60 & 1.28 & 0.71 \\
\hline $\mathrm{H}_{2} \mathrm{O}^{-}$ & 0.61 & 0.72 & 0.60 & 0.92 & 1.16 & 0.52 & 1.80 & 0.44 & 0.57 & 0.34 & 0.55 & 0.59 & 0.94 & 0.52 \\
\hline $\mathrm{P}_{2} \mathrm{O}_{5}$ & $\mathrm{tr}$ & 0.02 & 0.03 & $\mathrm{tr}$ & 0.04 & $\mathrm{tr}$ & 0.03 & 0.01 & 0.05 & 0.05 & 0.06 & 0.05 & 0.01 & 0.04 \\
\hline $\mathrm{C}^{2}$ & - & - & - & - & - & - & - & - & - & - & - & - & - & - \\
\hline Total & 100.51 & 100.54 & 100.68 & 100.49 & 100.58 & 100.01 & 100.01 & 100.55 & 100.06 & 100.53 & 100.50 & 100.43 & 99.77 & 100.05 \\
\hline
\end{tabular}


Table 1. (Continued).

\begin{tabular}{|c|c|c|c|c|c|c|c|c|c|c|c|c|c|c|c|}
\hline \multicolumn{8}{|c|}{$482 \mathrm{~B}$} & \multicolumn{8}{|c|}{$482 \mathrm{C}$} \\
\hline 20 & 20 & 21 & 21 & 22 & 22 & 24 & 24 & 10 & 11 & 11 & 12 & 13 & 14 & 15 & 15 \\
\hline 1 & 3 & 2 & 3 & 1 & 3 & 1 & 3 & 1 & 3 & 4 & 1 & 2 & 4 & 1 & 4 \\
\hline $120-127$ & $43-52$ & $9-18$ & $110-117$ & $97-105$ & $85-93$ & $106-115$ & $30-33$ & $107-115$ & $96-105$ & $83-91$ & $58-68$ & $80-90$ & $42-53$ & $1-10$ & $48-58$ \\
\hline 49.76 & 48.77 & 49.16 & 48.75 & 48.87 & 48.83 & 48.78 & 49.12 & 48.91 & 49.24 & 49.31 & 49.90 & 49.41 & 49.48 & 49.46 & 49.97 \\
\hline 1.57 & 1.49 & 1.44 & 1.57 & 57 & 1.53 & 1.87 & 1.91 & 1.87 & 1.87 & 1.87 & 1.36 & 1.40 & 1.36 & 1.36 & 1.36 \\
\hline 15.13 & 15.00 & 14.31 & 15.33 & 15.38 & 14.97 & 14.07 & 14.22 & 13.96 & 14.38 & $14: 00$ & 14.60 & 14.31 & 14.24 & $14: 40$ & 14.32 \\
\hline 2.41 & 3.82 & 3.46 & 3.81 & 3.23 & 3.55 & 4.29 & 3.50 & 3.73 & 3.29 & 3.42 & 2.83 & 3.36 & 2.98 & 3.29 & 2.42 \\
\hline 7.33 & 6.84 & 7.47 & 6.92 & 7.22 & 7.08 & 7.35 & 7.95 & 7.55 & 7.77 & 8.05 & 7.74 & 7.53 & 7.89 & 7.62 & 7.80 \\
\hline 0.17 & 0.17 & 0.18 & 0.18 & 0.18 & 0.18 & 0.17 & 0.18 & 0.18 & 0.17 & 0.17 & 0.17 & 0.17 & 0.17 & 0.18 & 0.17 \\
\hline 7.47 & 7.65 & 8.08 & 7.39 & 7.31 & 7.82 & 7.83 & 7.56 & 7.92 & 8.08 & 7.83 & 8.17 & 8.40 & 8.16 & 8.26 & 7.91 \\
\hline 12.18 & 12.44 & 12.44 & 12.32 & 12.56 & 12.44 & 11.62 & 11.83 & 11.86 & 11.96 & 11.61 & 12.33 & 12.05 & 12.54 & 12.22 & 12.69 \\
\hline 2.43 & 2.61 & 2.41 & 2.61 & 2.70 & 2.50 & & & 2.61 & 2.51 & 2.51 & 2.32 & 2.41 & 2.32 & 2.32 & 2.32 \\
\hline 0.17 & 0.05 & 0.10 & 0.10 & 0.05 & 0.05 & 0.10 & 0.10 & 0.15 & 0.15 & 0.10 & 0.1 & 0.10 & 0.1 & 0.10 & 0.10 \\
\hline 0.76 & 0.46 & 0.36 & 0.31 & 0.20 & 0.55 & 0.32 & 0.33 & 0.41 & 0.37 & 0.55 & 0.30 & 0.41 & 0.54 & 0.43 & 0.31 \\
\hline 0.34 & 0.77 & 0.58 & 0.82 & 0.74 & 0.79 & 0.88 & 0.72 & & 0.58 & & 0.54 & 0.58 & 0.50 & 0.56 & 0.58 \\
\hline 0.08 & 0.01 & 0.03 & tr & 0.01 & 0.01 & tr & - & 0.01 & 0.02 & 0.01 & $\operatorname{tr}$ & 0.01 & 0.02 & 0.01 & $\mathrm{tr}$ \\
\hline- & - & - & - & - & - & - & - & - & - & - & - & - & - & - & - \\
\hline- & - & - & - & - & - & - & - & - & - & - & - & - & 0.12 & - & - \\
\hline 100.25 & 100.08 & 100.02 & 100.11 & 100.02 & 100.30 & 100.10 & 100.12 & 100.13 & 100.39 & 100.07 & 100.36 & 100.14 & 100.42 & 100.21 & 99.95 \\
\hline
\end{tabular}

Table 1. (Continued).

\begin{tabular}{|c|c|c|c|c|c|c|c|c|c|c|c|c|c|c|c|}
\hline & & & 483 & & & & & & & & 483 & & & & \\
\hline 20 & 21 & 22 & 23 & 25 & 26 & 26 & 4 & 7 & 8 & 12 & 13 & 17 & 19 & 22 & 25 \\
\hline 1 & 2 & 4. & 2 & 1 & 2 & 3 & 5 & 2 & 3 & 1 & 3 & 1 & 2 & 2 & 1 \\
\hline $118-127$ & $43-51$ & $117-127$ & $92-103$ & $48-58$ & $140-149$ & $98-108$ & $13-23$ & $8-19$ & $26-35$ & $40-46$ & $64-74$ & $138-146$ & $21-30$ & $13-23$ & $103-114$ \\
\hline 48.63 & 48.69 & 48.16 & 48.23 & 47.92 & 48.11 & 48.70 & 48.57 & 48.87 & 46.63 & 48.45 & 48.23 & 48.28 & 47.65 & 48.64 & 48.72 \\
\hline 1.94 & 2.12 & 1.93 & 1.99 & 2.56 & 2.36 & 2.51 & 1.34 & 1.65 & 1.03 & 1.87 & 2.09 & 2.32 & 2.39 & 1.97 & 2.04 \\
\hline 13.22 & 13.06 & 14.19 & 15.49 & 13.75 & 13.54 & 13.06 & 14.93 & 14.61 & 15.76 & 15.49 & 13.21 & 13.64 & 13.66 & 14.56 & 14.45 \\
\hline 4.49 & 4.52 & 4.40 & 5.18 & 4.71 & 5.34 & 5.64 & 2.76 & 3.15 & 4.34 & 2.87 & 7.37 & 4.64 & 6.26 & 3.50 & 3.19 \\
\hline 8.93 & 9.11 & 7.58 & 6.22 & 8.65 & 8.55 & 8.77 & 7.44 & 7.54 & 6.35 & 7.99 & 7.06 & 8.96 & 7.89 & 8.64 & 8.42 \\
\hline 0.17 & 0.17 & 0.18 & 0.17 & 0.19 & 0.19 & 0.19 & 0.16 & 0.17 & 0.17 & 0.20 & 0.19 & 0.20 & 0.18 & 0.18 & 0.18 \\
\hline 7.70 & 7.48 & 7.33 & 7.44 & 6.96 & 6.87 & 7.03 & 8.73 & 7.74 & 10.26 & 7.43 & 7.42 & 7.13 & 7.05 & 7.68 & 7.84 \\
\hline 10.50 & 11.55 & 12.02 & 10.96 & 11.29 & 11.01 & 10.84 & 12.21 & 12.19 & 11.59 & 11.71 & 11.06 & 11.04 & 10.96 & 11.71 & 11.19 \\
\hline 2.70 & 2.70 & 2.57 & 2.85 & 2.85 & & 2.61 & 2.41 & 2.61 & 2.13 & 2.51 & 2.70 & 2.7 & 2.94 & 2.70 & 2.59 \\
\hline 0.13 & 0.13 & 0.13 & 0.13 & 0.17 & 0.13 & 0.15 & 0.10 & 0.15 & 0.10 & 0.18 & 0.20 & 0.20 & 0.15 & 0.15 & 0.18 \\
\hline 0.29 & 0.13 & 0.59 & 0.54 & 0.32 & 0.53 & 0.37 & 0.57 & 0.42 & 1.50 & 0.30 & 0.33 & 0.72 & 0.36 & 0.27 . & 0.36 \\
\hline 0.63 & 0.59 & 0.74 & 1.08 & 0.54 & 0.77 & 0.68 & 0.50 & 0.58 & 0.59 & 0.54 & 0.66 & 0.55 & 0.59 & 0.57 & 0.80 \\
\hline 0.12 & 0.02 & tr & tr & $\operatorname{tr}$ & $\operatorname{tr}$ & tr & tr & tr & tr & tr & tr & tr & tr & tr & tr \\
\hline - & - & - & 0.15 & - & - & - & - & - & - & - & - & - & - & - & - \\
\hline- & - & - & - & - & - & - & - & - & - & - & - & - & - & - & - \\
\hline 100.45 & 100.27 & 99.82 & 100.43 & 99.91 & 100.25 & 100.55 & 99.72 & 99.68 & 100.45 & 99.54 & 100.52 & 100.38 & 100.08 & 100.57 & 99.96 \\
\hline
\end{tabular}

Table 1. (Continued).

\begin{tabular}{|c|c|c|c|c|c|c|c|c|c|c|c|c|c|}
\hline \multicolumn{14}{|c|}{$485 \mathrm{~A}$} \\
\hline 23 & 24 & 25 & 29 & 30 & 30 & 31 & 33 & 34 & 35 & 35 & 38 & 39 & 39 \\
\hline 3 & 2 & 1 & 3 & 1 & 2 & 1 & 2 & 2 & 1 & 5 & 2 & 3 & 4 \\
\hline $60-72$ & $77-87$ & $63-74$ & $118-130$ & $121-134$ & $116-129$ & $98-119$ & $7-19$ & $78-89$ & $21-31$ & $48-62$ & $104-119$ & $47-57$ & $105-115$ \\
\hline 49.12 & 48.59 & 48.73 & 48.92 & 48.51 & 49.52 & 48.18 & 48.13 & 48.73 & 48.81 & 48.59 & 48.16 & 48.31 & 48.38 \\
\hline 2.16 & 1.95 & 2.08 & 2.12 & 2.71 & 2.04 & 1.95 & 2.29 & 1.94 & 1.96 & 1.95 & 2.12 & 2.26 & 1.93 \\
\hline 14.11 & 14.55 & 13.81 & 12.30 & 11.12 & 13.60 & 13.81 & 13.44 & 12.89 & $13: 83$ & 13.49 & 14.26 & 14.29 & 13.84 \\
\hline 4.06 & 3.58 & 4.48 & 4.16 & 6.06 & 3.19 & 3.64 & 4.00 & 4.04 & 3.09 & 3.18 & 3.04 & 3.33 & 3.26 \\
\hline 8.30 & 8.72 & 8.65 & 8.86 & 9.74 & 7.39 & 8.87 & 9.43 & 8.55 & 8.65 & 8.83 & 8.83 & 8.84 & 8.91 \\
\hline 0.18 & 0.17 & 0.20 & 0.17 & 0.17 & 0.14 & 0.16 & 0.17 & 0.14 & 0.14 & 0.14 & 0.16 & 0.14 & 0.14 \\
\hline 6.83 & 7.46 & 6.92 & 7.75 & 7.23 & 8.29 & 8.33 & 7.99 & 8.21 & 7.86 & 7.75 & 7.84 & 6.83 & 8.07 \\
\hline 11.65 & 11.22 & 11.68 & 11.57 & 10.39 & 12.64 & 11.21 & 10.40 & 11.60 & 11.81 & 12.24 & 11.11 & 10.94 & 11.52 \\
\hline 2.61 & 2.61 & 2.60 & 2.70 & 2.70 & 2.49 & 2.49 & 2.51 & 2.41 & 2.49 & 2.49 & 2.49 & 2.70 & 2.60 \\
\hline 0.12 & 0.24 & 0.15 & 0.15 & 0.15 & 0.15 & 0.20 & 0.24 & 0.12 & 0.10 & 0.10 & 0.15 & 0.10 & 0.10 \\
\hline 0.55 & 0.81 & 0.68 & 0.68 & 0.50 & 0.40 & 0.60 & 0.73 & 0.65 & 0.72 & 0.62 & 1.15 & 0.72 & 0.73 \\
\hline 0.50 & 0.52 & 0.62 & 0.73 & 0.95 & 0.34 & 0.79 & 0.86 & 0.87 & 0.66 & 0.71 & 0.90 & 1.17 & 0.90 \\
\hline 0.04 & 0.04 & 0.04 & 0.01 & 0.14 & 0.04 & 0.02 & 0.01 & 0.03 & 0.01 & 0.01 & 0.02 & 0.01 & 0.01 \\
\hline- & 0.20 & - & - & - & - & - & - & - & - & - & - & - & - \\
\hline- & - & - & - & - & - & - & - & - & - & 0.14 & - & - & - \\
\hline 100.23 & 100.66 & 100.64 & 100.12 & 100.37 & 100.23 & 100.25 & 100.20 & 100.38 & 100.13 & 100.24 & 100.23 & 99.64 & 100.39 \\
\hline
\end{tabular}




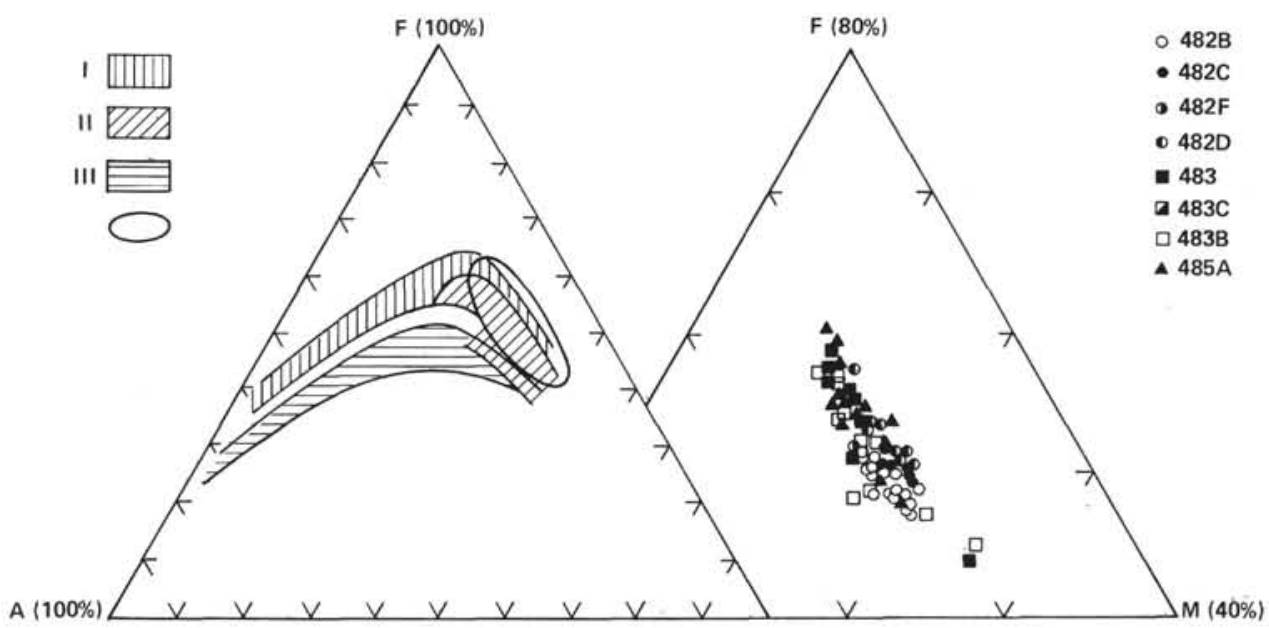

Figure 2. AFM diagrams comparing Leg 65 basalt compositions (right) with compositional fields for (I) tholeiitic basalts, (II) high aluminum basalts, and (III) alkaline olivine basalts (left); ellipse shows compositional field of Leg 65 basalts. $\mathrm{A}=\mathrm{Na}_{2} \mathrm{O}+\mathrm{K}_{2} \mathrm{O} ; \mathrm{F}=\mathrm{FeO}+\mathrm{Fe}_{2} \mathrm{O}_{3} ; \mathrm{M}=\mathrm{MgO}$. (Values shown in wt.\%.)

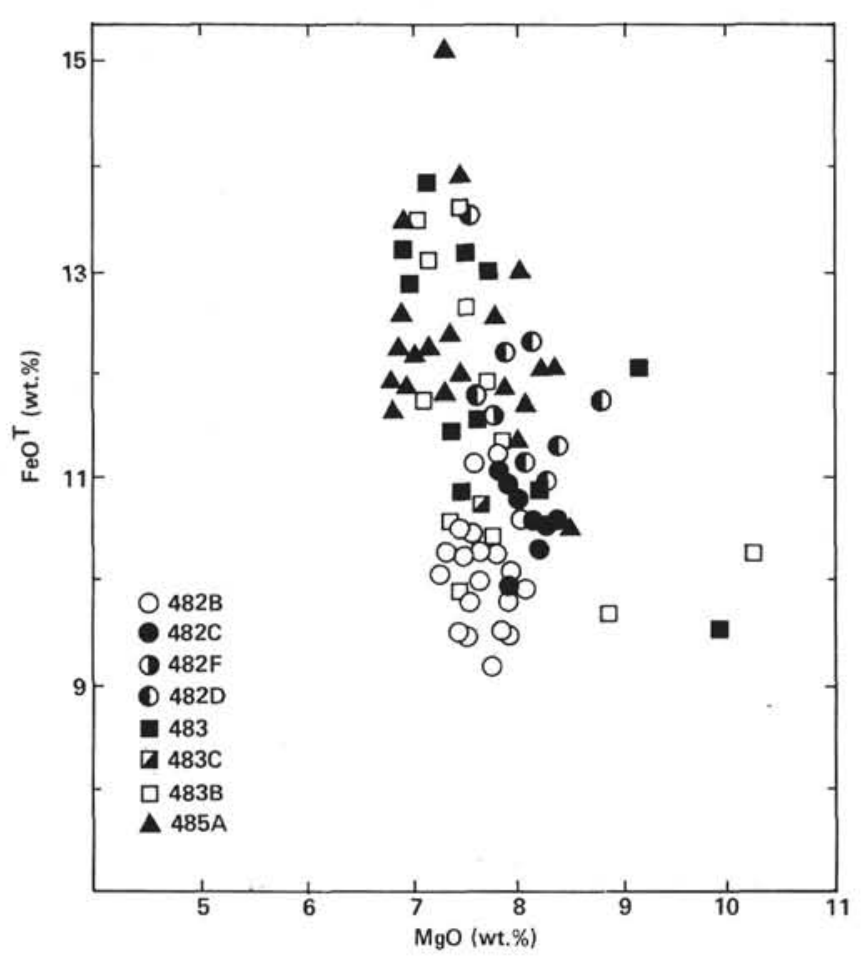

Figure 3. $\mathrm{FeO}^{\mathrm{T}}$ vs. $\mathrm{MgO}$ diagram for Leg 65 basalts.

differences in the overall concentration of rare-earth elements were less significant during the earlier stages of volcanism that are closer to the primary melt.

This allows us to conclude that primary magmatic melts giving rise to basalts at these sites formed at the expense of partial melting of compositionally similar mantle material under similar T-P conditions.

What was the composition of the mantle material from which the basalts formed? Variation diagrams of standardized ratios of rare-earth elements have been compiled comparing concentrations of REE in Leg 65 basalts to those in abyssal oceanic tholeiite, chondrite, plagioclase-bearing lherzolite, clinopyroxene, and plagio-

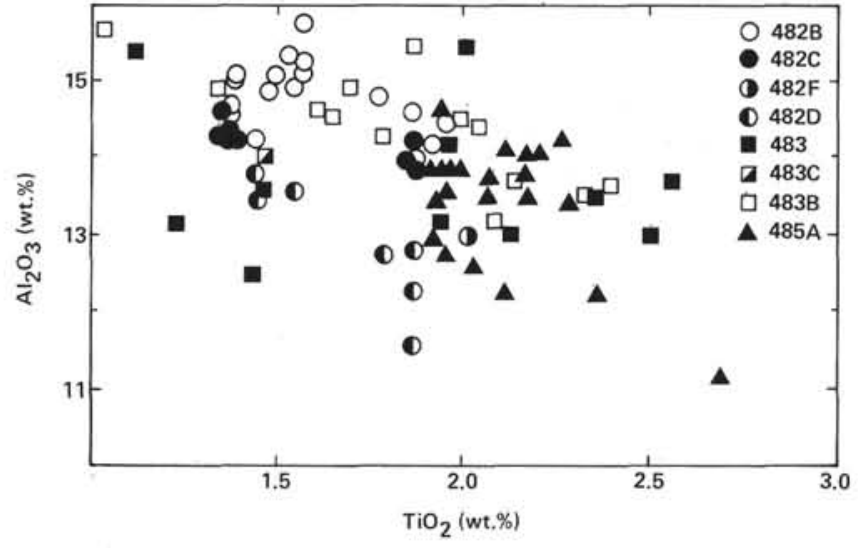

Figure 4. $\mathrm{Al}_{2} \mathrm{O}_{3}$ vs. $\mathrm{TiO}_{2}$ diagram for $\mathrm{Leg} 65$ basalts.

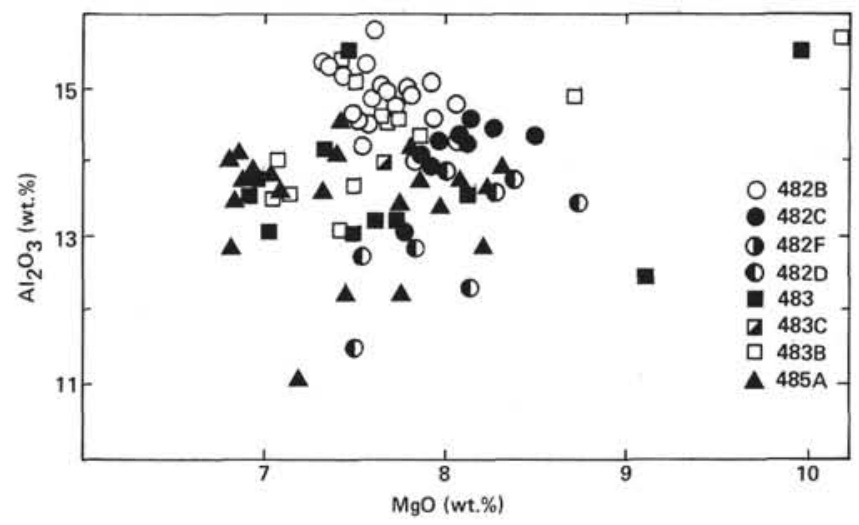

Figure 5. $\mathrm{Al}_{2} \mathrm{O}_{3}$ vs. $\mathrm{MgO}$ diagram for Leg 65 basalts.

clase (Fig. 13). It follows from examination of these diagrams that the character of distribution of rare-earth elements in Leg 65 basalts is tentatively the same in all sites and is very close to their distribution of the median oceanic tholeiite. Considering the distribution of rareearth elements, plagioclase-containing lherzolite from the supposed mantle substratum is most similar to the 


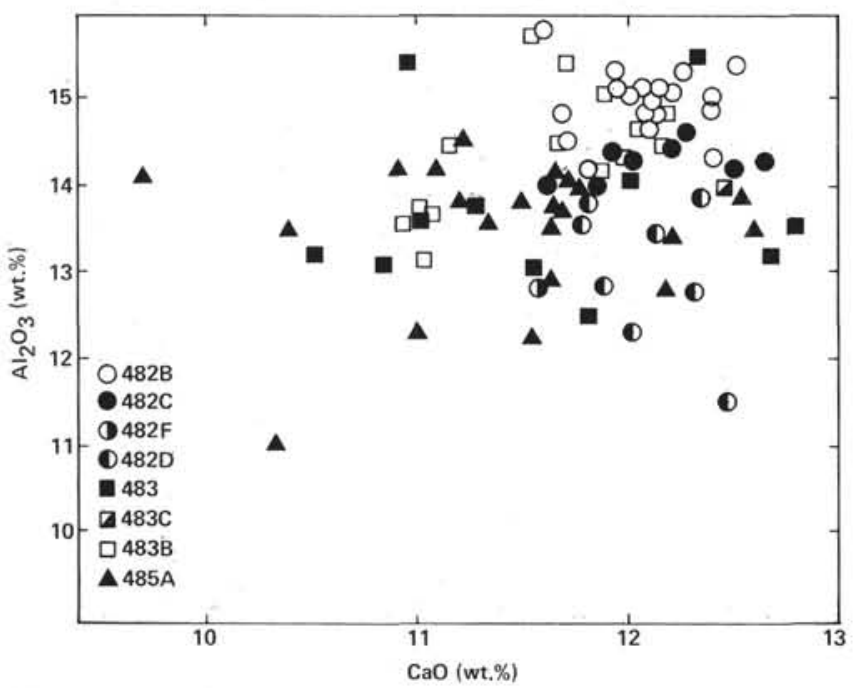

Figure 6. $\mathrm{Al}_{2} \mathrm{O}_{3}$ vs. $\mathrm{CaO}$ diagram for Leg 65 basalts.

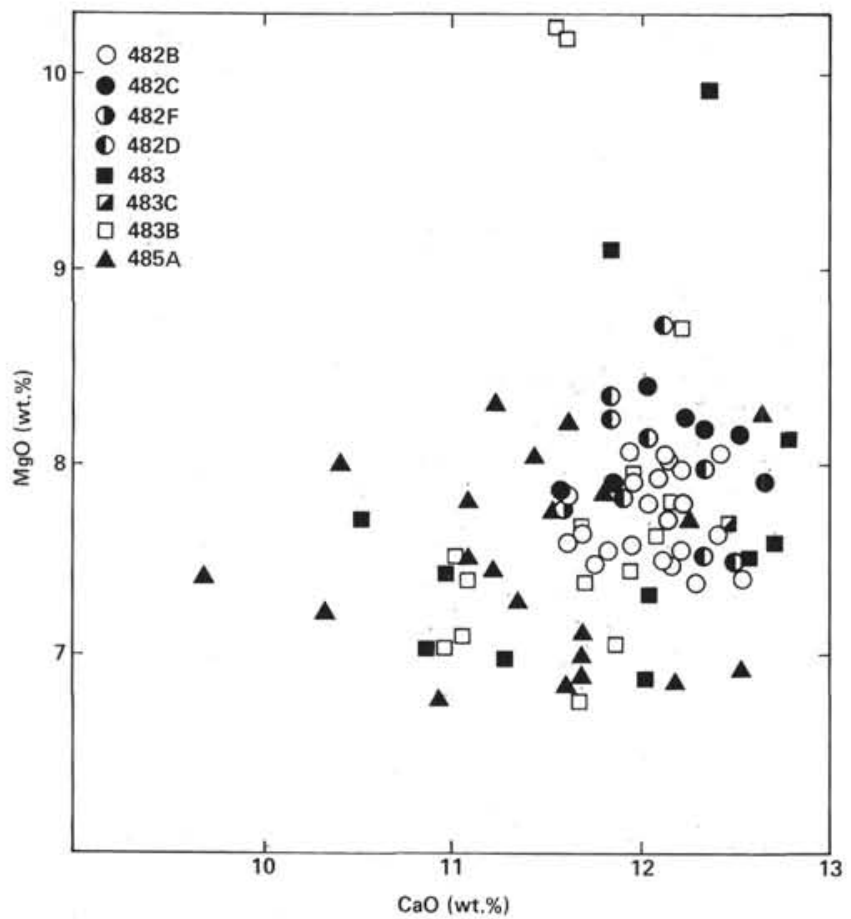

Figure 7. $\mathrm{MgO}$ vs. $\mathrm{CaO}$ diagram for Leg 65 basalts.

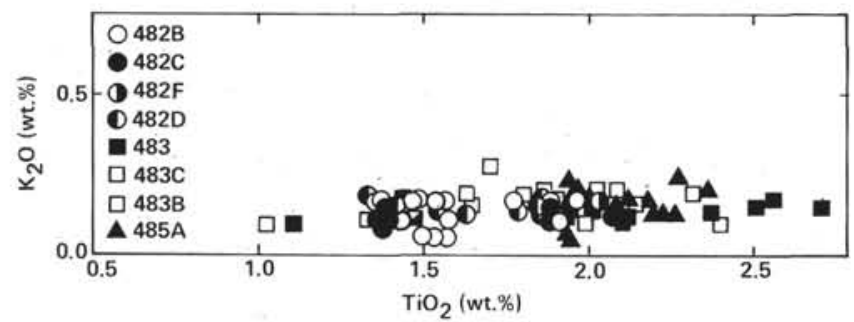

Figure 8. $\mathrm{K}_{2} \mathrm{O}$ vs. $\mathrm{TiO}_{2}$ diagram for $\mathrm{Leg} 65$ basalts.

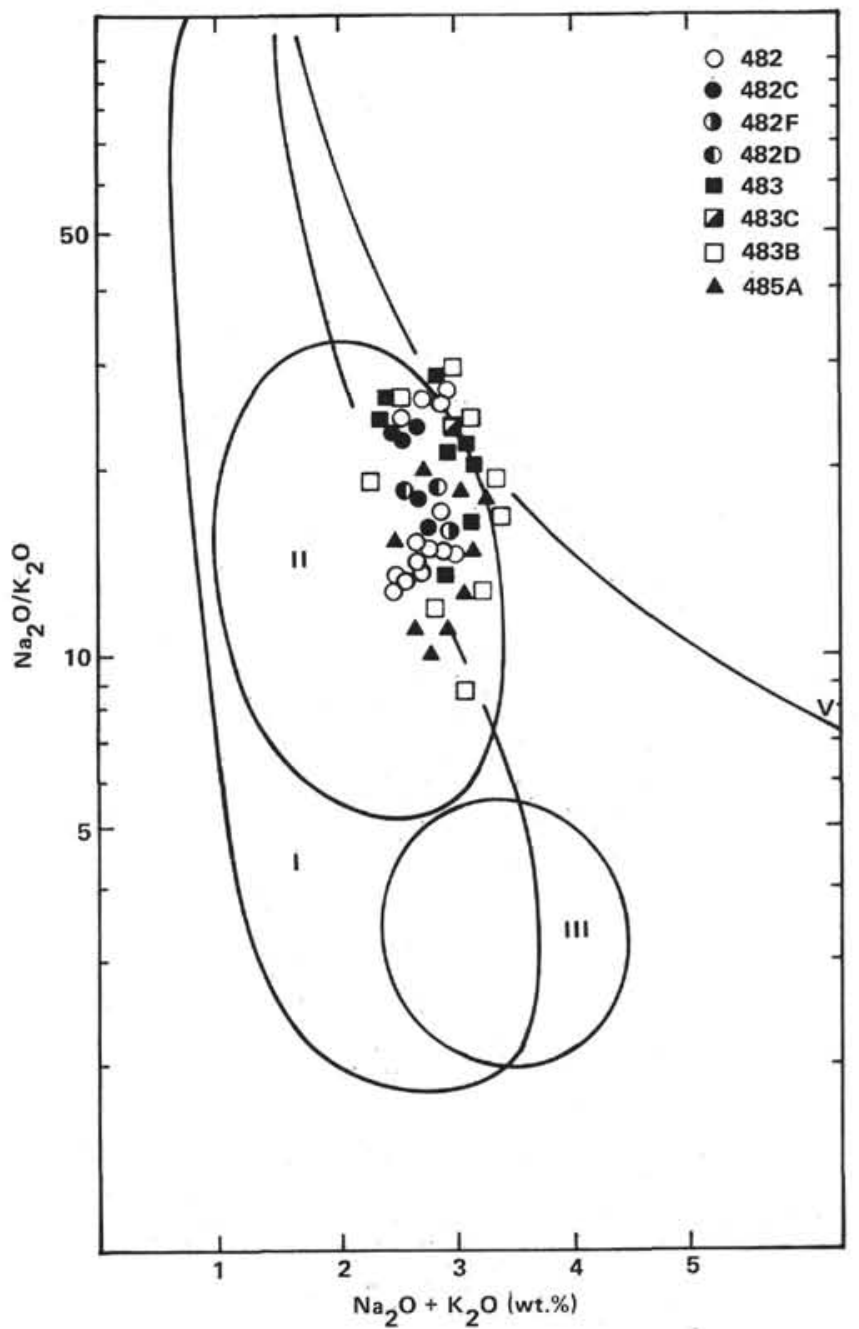

Figure 9. $\mathrm{Na}_{2} \mathrm{O} / \mathrm{K}_{2} \mathrm{O}$ vs. $\mathrm{Na}_{2} \mathrm{O}+\mathrm{K}_{2} \mathrm{O}$ (Miyashiro diagram) for Leg 65 basalts. Also shown are compositional fields for (I) Icelandic tholeiites; (II) abyssal oceanic tholeiites; and (III) alkaline olivine basalts on Atlantic islands; Line V separates fresh rocks from those which display secondary alteration.

Leg 65 basalts, whereas clinopyroxene is the most similar mineral.

We can assume that Leg 65 basalts resulted from partial melting of plagioclase-bearing lherzolite, involving mainly clinopyroxene, possibly admixed with small amounts of plagioclase. Variations in compositions of primary melts at various sites were controlled by different degrees of partial melting, but not by differences in the composition of the mantle substratum.

\section{CONCLUSIONS}

Two structural types are found among volcanic rocks penetrated by drilling during DSDP Leg 65: pillow basalts and massive crystalline basalts. Both structural types contain aphyric and sparsely phyric plagioclase and plagioclase-olivine varieties. Volumetrically, aphyric rocks are insignificant. By chemical composition, all Leg 65 basalts are abyssal oceanic tholeiites with a poorly manifested Fenner trend of differentiation with accumulation of iron. We managed to establish insignificant 


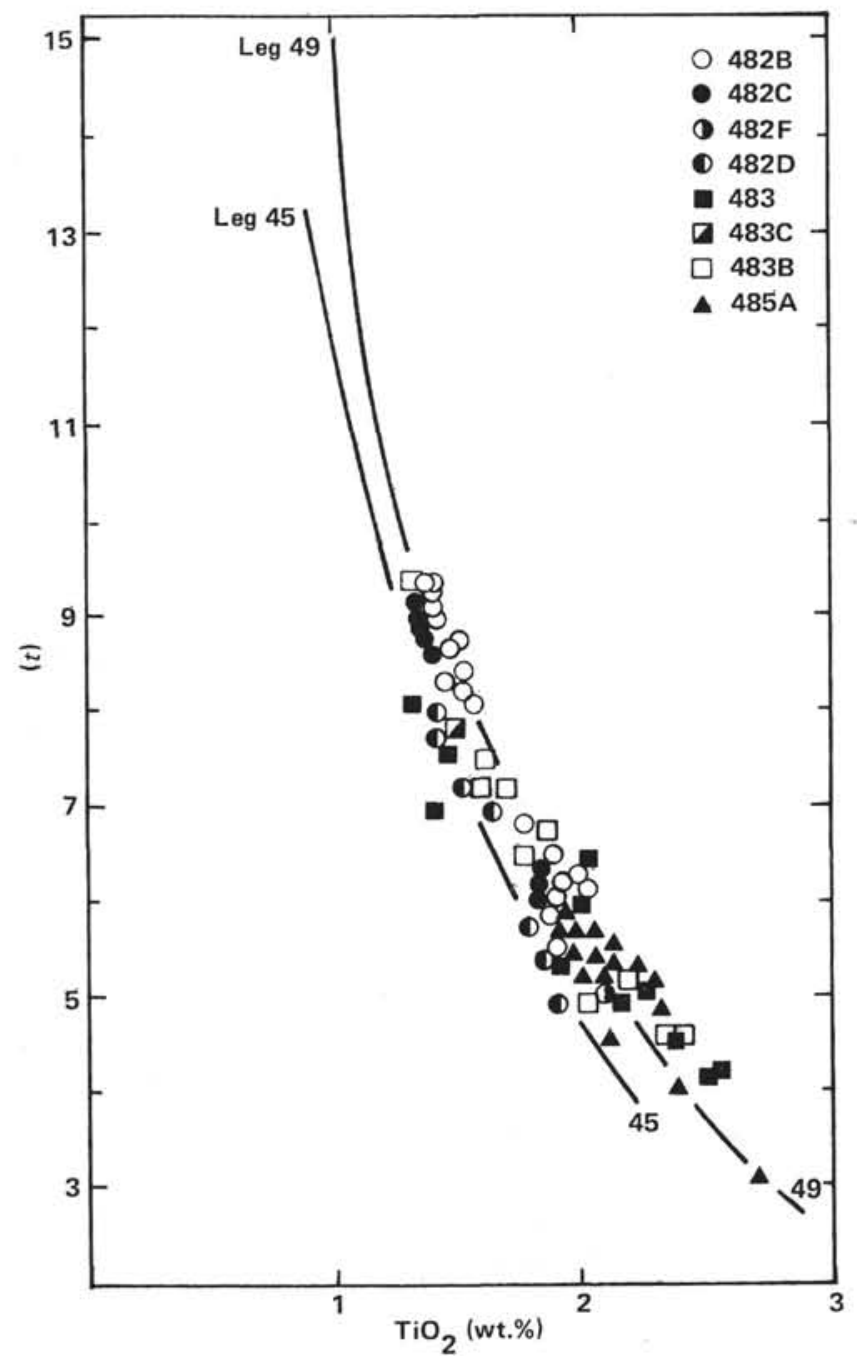

Figure 10. $\mathrm{TiO}_{2}$ vs. $t$ diagram for Leg 65 basalts; where $t=\mathrm{Al}_{2} \mathrm{O}_{3}-$ $\mathrm{Na}_{2} \mathrm{O} / \mathrm{TiO}_{2}$,

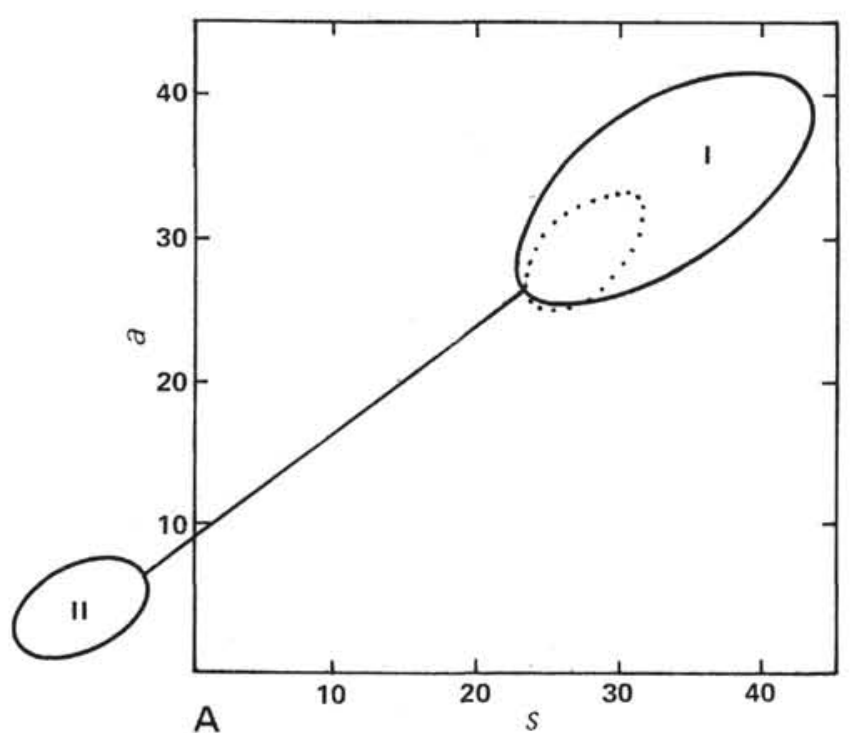

variations in chemistry of basalts penetrated at various sites in the Gulf of California. These variations of the basalt composition are the result of different degrees of partial melting to form the primary melt, but are not due to subsequent crystallization differentiation. The formation of primary melts took place at depths not exceeding 25 to $30 \mathrm{~km}$. Plagioclase lherzolite is assumed to be the parental mantle material which melted to form the basalts. The character of distribution of rare-earth elements in the basalts studied does not contradict these conclusions.

\section{ACKNOWLEDGMENTS}

The authors express their deep gratitude to Academician A. V. Peive, Director of the Geological Institute of the USSR Academy of Sciences, and to Dr. David G. Moore and Dr. M. N. A. Peterson of the Deep Sea Drilling Project for the opportunity to participate on Leg 65 . We are pleased to mention the friendly and creative conditions on board the Glomar Challenger promoted by Captain Dill and the ship's officers and crew. We also thank G. F. Galkovskaya, N. L. Kalashnikova, G. I. Karaseva, and V. F. Rychkova who carried out the chemical analyses, and our colleagues S. M. Lyapunov and A. M. Shshilnikov for their advice during the course of this investigation.

\section{REFERENCES}

Balashov, Yu. A., 1976. Geochemistry of Rare-Earth Elements: Moscow (Izdat. Nauka), p. 267 (Russian).

Green, D. H., and Ringwood, A. E., 1967. The genesis of basaltic magmas. Contrib. Mineral. Petrol., 15:103-190.

Hall, J. M., and Robinson, P. T., 1979. Deep crustal drilling in the North Atlantic ocean. Science, 204:573-566.

MacDonald, G., A., 1968. Composition and origin of Hawaiian lavas. Contrib. Hawaii Inst. Geophys., pp. 477-522.

Zaitsev, E. I., Sotskov, Yu. P., and Reznikov, R. S., 1978. NeutronActivation Analysis of Mountain Rocks for Rare Elements: Moscow (Izdat. Nedra), p. 100. (Russian)

Zolotarev, B. P., 1979. Petrology of basalts of the recent ocean relative to their tectonic position. Geotektonika, 1:22-35. (Russian)

Zolotarev, B. P., Choporov, D. Ya., and Voitov, G. I., 1979. Petrochemistry of basalts and distribution of organic gases: Holes 407, 408, 409, 410A, 411, 412, and 413, DSDP, Leg 49. In Luyendyk, B. P., Cann, J. R., et al., Init. Repts. DSDP, 49: Washington (U.S. Govt. Printing Office), pp. 727-744.

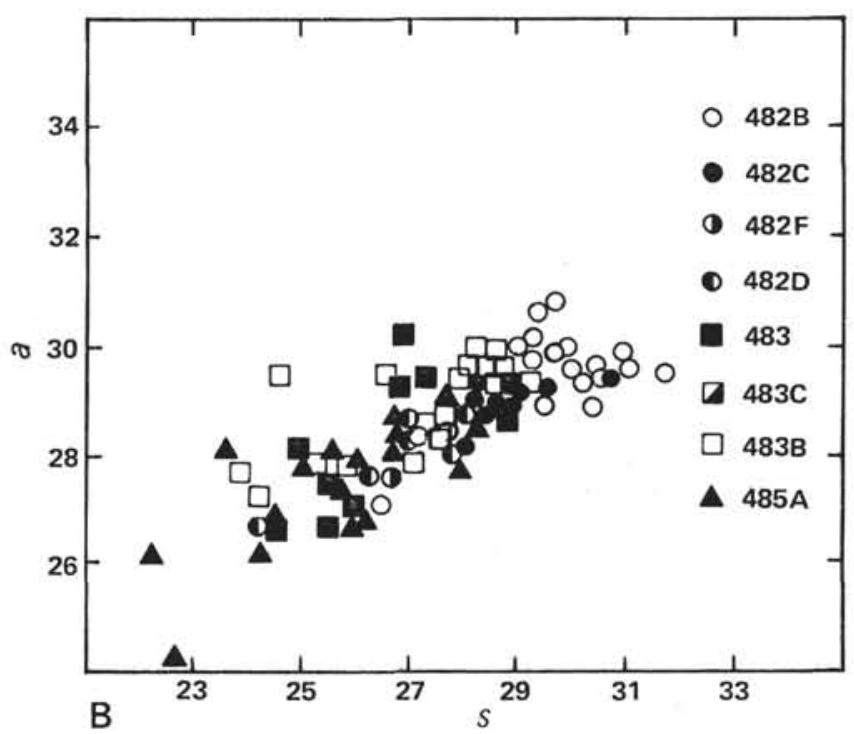

Figure 11. Dmitriev diagrams comparing Leg 65 basalt compositions (B) with compositional fields for (I) abyssal oceanic tholeiites in the Atlantic and (II) oceanic lherzolites (A); dotted ellipse in A shows compositional field of Leg 65 basalts. $\left(a=\mathrm{Al}_{2} \mathrm{O}_{3}+\mathrm{CaO}+\mathrm{Na}_{2} \mathrm{O}+\mathrm{K}_{2} \mathrm{O} ; s=\mathrm{SiO}_{2}\right.$ $-\left[\mathrm{MgO}+\mathrm{Fe}_{2} \mathrm{O}_{3}+\mathrm{FeO}+\mathrm{TiO}_{2}+\mathrm{MnO}\right]$.) 


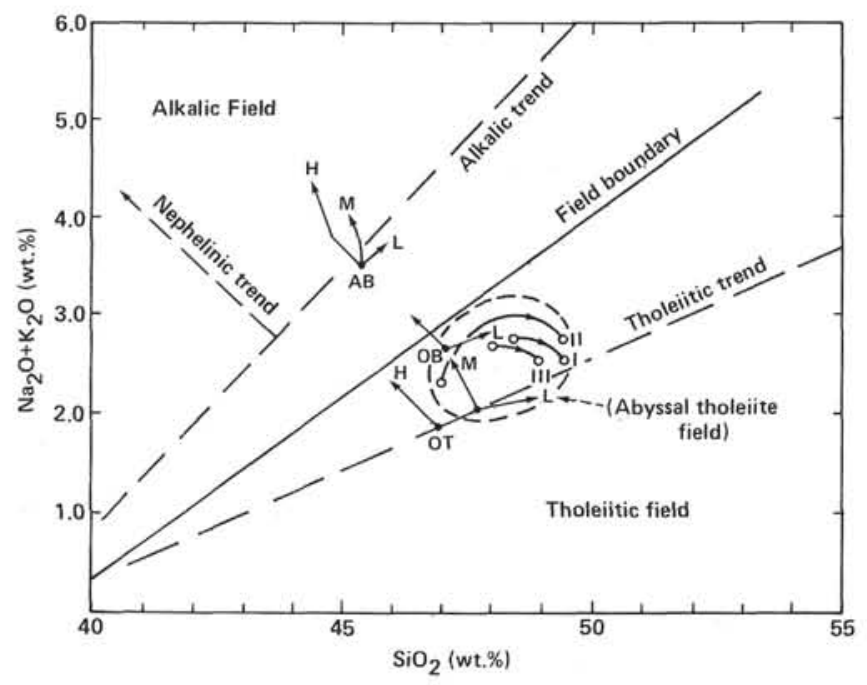

Figure 12. Alkali-silica diagram showing differentiation trends at (I) Site 482 , (II) Site 483 , and (III) Site 485 . AB = composition of primary melt for alkaline olivine basalts; OT composition of primary melt for oceanic tholeiites; $\mathrm{OB}=$ composition of primary melt for olivine basalts (mixture of OT and $\mathrm{AB}$ ); $\mathrm{H}, \mathrm{M}$, and $\mathrm{L}$ show paths taken by melts crystallizing under relatively high (13-18 kb), intermediate $(9 \mathrm{~kb})$, and low pressures (data from Green and Ringwood, 1967).

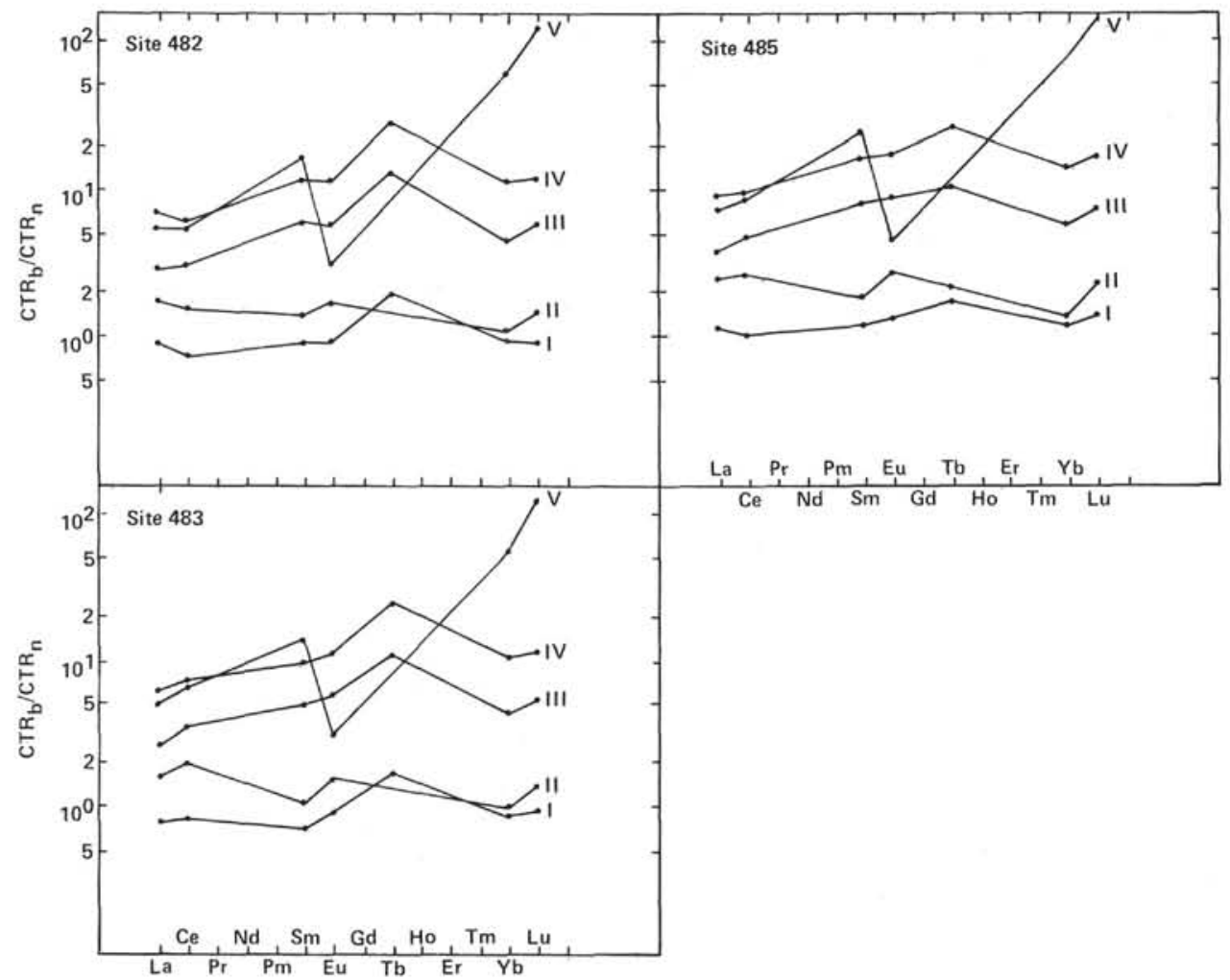

Figure 13. Rare-earth elements in basalts at the various sites, normalized to (I) average abyssal oceanic tholeiite; (II) clinopyroxene; (III) plagioclase-bearing lherzolite; (IV) the silicate phase in chondrites; (V) plagioclase. $C T R_{b} / C T R_{n}=$ ratio of concentration of rare-earth elements in basalts to concentration of rare-earth elements in the various rocks, minerals, and chondrites (from Yu. A. Balashov, 1976). 
Table 2. Rare-earth element abundances in basalts from Sites 482,483 , and $485 .^{\mathrm{a}}$

\begin{tabular}{|c|c|c|c|c|c|c|c|c|c|c|c|c|c|}
\hline \multirow{4}{*}{$\begin{array}{l}\text { Hole } \\
\text { Core } \\
\text { Section } \\
\text { Interval }(\mathrm{cm})\end{array}$} & \multicolumn{13}{|c|}{$482 B$} \\
\hline & 11 & 12 & 13 & 14 & 14 & 14 & 14 & 15 & 15 & 15 & 16 & 16 & 18 \\
\hline & 1 & 1 & 1 & 1 & 1 & 2 & 3 & 1 & 2 & 3 & 1 & 5 & 1 \\
\hline & $77-85$ & $107-113$ & $54-61$ & $12-20$ & $101-106$ & $94-101$ & $109-119$ & $94-100$ & $120-129$ & $20-30$ & $41-50$ & $39-49$ & $49-57$ \\
\hline $\mathrm{La}$ & 4.0 & 4.2 & 3.9 & 3.8 & 4.4 & 3.0 & 3.0 & 2.6 & 2.4 & 4.0 & 2.6 & 3.5 & 3.1 \\
\hline $\mathrm{Ce}$ & 9.5 & ND & ND & ND & ND & 9.2 & ND & 9.7 & ND & ND & ND & 7.8 & ND \\
\hline $\mathrm{Sm}$ & 4.7 & 4.8 & 4.6 & 5.1 & 3.8 & 3.9 & 4.1 & 3.8 & 3.0 & 3.3 & 3.1 & 3.4 & 3.2 \\
\hline Eu & 1.6 & 1.25 & 1.2 & 1.5 & 1.25 & 1.7 & 1.0 & 1.5 & 1.25 & 1.1 & 0.99 & 1.4 & 1.8 \\
\hline $\mathrm{Tb}$ & 2.88 & ND & ND & ND & ND & 1.5 & ND & 2.53 & ND & ND & ND & 1.15 & ND \\
\hline $\mathrm{Yb}$ & 3.2 & 3.7 & 3.4 & 3.6 & 3.0 & 2.9 & 2.9 & 3.0 & 3.6 & 3.2 & 2.3 & 2.7 & 2.8 \\
\hline $\mathrm{Lu}$ & 0.63 & 0.61 & 0.6 & 0.71 & 0.55 & 0.54 & 0.40 & 0.58 & 0.61 & 0.42 & 0.46 & 0.47 & 0.62 \\
\hline
\end{tabular}

Note: $\mathrm{ND}=$ not determined.

a Values shown in ppm.

Table 2, (Continued).

\begin{tabular}{|c|c|c|c|c|c|c|c|c|c|c|c|c|c|}
\hline \multirow{2}{*}{$\begin{array}{l}\text { Hole } \\
\text { Core }\end{array}$} & \multicolumn{9}{|c|}{$482 \mathrm{D}$} & \multirow{2}{*}{$\frac{482 \mathrm{~F}}{5}$} & \multicolumn{3}{|c|}{483} \\
\hline & 15 & 8 & 9 & 9 & 10 & 11 & 12 & 12 & 13 & & 14 & 15 & 16 \\
\hline Section & 4 & 1 & 1 & 2 & 1 & 1 & 1 & 3 & 1 & 1 & 1 & 1 & 1 \\
\hline Interval $(\mathrm{cm})$ & $48-58$ & $115-124$ & $59-68$ & $130-139$ & $133-139$ & $110-118$ & $80-87$ & $104-108$ & $59-70$ & $100-108$ & $13-21$ & $121-130$ & $71-83$ \\
\hline $\mathrm{La}$ & 3.7 & 4.0 & 5.0 & 4.3 & 4.8 & 3.1 & 2.5 & 3.2 & 3.6 & 3.8 & 3.5 & 3.7 & 2.6 \\
\hline $\mathrm{Ce}$ & ND & ND & 11 & ND & ND & 8.7 & ND & ND & ND & 11 & ND & ND & 6.7 \\
\hline Sm & 3.1 & 4.2 & 4.8 & 4.8 & 4.8 & 3.9 & 3.8 & 3.2 & 3.7 & 4.8 & 3.1 & 3.4 & 3.5 \\
\hline $\mathrm{Eu}$ & 1.3 & 1.6 & 1.7 & 1.8 & 1.1 & 1.4 & 1.2 & 1.2 & 1.9 & 1.9 & 1.4 & 1.2 & 1.5 \\
\hline $\mathrm{Tb}$ & ND & ND & 2.07 & ND & ND & 1.96 & ND & ND & ND & 2.88 & ND & ND & 1.61 \\
\hline $\mathrm{Yb}$ & 2.2 & 3.3 & 4.1 & 4.3 & 3.6 & 3.1 & 3.2 & 3.2 & 3.3 & 3.6 & 3.1 & 3.5 & 2.6 \\
\hline Lu & 0.55 & 0.68 & 0.67 & 0.7 & 0.73 & 0.53 & 0.56 & 0.66 & 0.49 & 0.61 & 0.62 & 0.63 & 0.57 \\
\hline
\end{tabular}

Table 2. (Continued).

\begin{tabular}{|c|c|c|c|c|c|c|c|c|c|c|c|c|c|c|}
\hline \multirow{4}{*}{$\begin{array}{l}\text { Hole } \\
\text { Core } \\
\text { Section } \\
\text { Interval }(\mathrm{cm})\end{array}$} & \multicolumn{7}{|c|}{$483 \mathrm{~B}$} & \multirow{2}{*}{$\frac{483 C}{4}$} & \multicolumn{6}{|c|}{$485 \mathrm{~A}$} \\
\hline & 22 & 25 & 27 & 27 & 30 & 32 & 32 & & 11 & 11 & 12 & 13 & 14 & 18 \\
\hline & 2 & 1 & 2 & 3 & 1 & 1 & 3 & 3 & 3 & 1 & 1 & 1 & 1 & 1 \\
\hline & $13-23$ & $103-114$ & $10-21$ & $15-25$ & $70-78$ & $110-119$ & $33-40$ & $3-14$ & $140-148$ & $13-26$ & $21-33$ & $35-42$ & $43-56$ & $9-20$ \\
\hline $\mathrm{La}$ & 3.5 & 3.9 & 4.4 & 4.4 & 4.5 & 3.9 & 4.5 & 2.7 & 4.3 & 6.8 & 4.6 & 3.9 & 3.5 & 5.1 \\
\hline $\mathrm{Ce}$ & ND & ND & 14 & ND & ND & ND & ND & 9.4 & ND & ND & ND & ND & 12 & ND \\
\hline Sm & 4.2 & 4.4 & 4.7 & 4.9 & 4.4 & 3.7 & 4.0 & 3.2 & 5.0 & 5.3 & 5.2 & 4.9 & 4.9 & 5.0 \\
\hline $\mathrm{Eu}$ & 1.8 & 1.9 & 2.0 & 2.0 & 1.8 & 1.9 & 1.8 & 1.6 & 2.0 & 2.0 & 2.8 & 2.0 & 1.8 & 2.1 \\
\hline $\mathrm{Tb}$ & ND & ND & 1.61 & ND & ND & ND & ND & 1.15 & ND & ND & ND & ND & 1.84 & ND \\
\hline $\mathrm{Yb}$ & 3.8 & 4.2 & 3.9 & 4.1 & 3.6 & 3.7 & 3.1 & 2.7 & 4.0 & 5.8 & 5.3 & 4.5 & 4.4 & 5.3 \\
\hline $\mathrm{Lu}$ & 0.67 & 0.81 & 0.81 & 0.95 & 0.7 & 0.8 & 0.57 & 0.68 & 0.88 & 1.15 & 0.93 & 1.0 & 0.97 & 0.79 \\
\hline
\end{tabular}


Table 2. (Continued).

\begin{tabular}{|c|c|c|c|c|c|c|c|c|c|c|c|c|c|c|c|}
\hline \multicolumn{9}{|c|}{$482 B$} & \multicolumn{7}{|c|}{$482 \mathrm{C}$} \\
\hline 18 & 20 & 20 & 21 & 21 & 22 & 22 & 24 & 24 & 10 & 11 & 11 & 12 & 13 & 14 & 15 \\
\hline 2 & 1 & 3 & 2 & 3 & 1 & 3 & 1 & 3 & 1 & 3 & 4 & 1 & 2 & 4 & 1 \\
\hline $44-51$ & $120-127$ & $43-52$ & $9-18$ & $110-117$ & $97-95$ & $85-93$ & $106-115$ & $30-39$ & $107-115$ & $96-105$ & $83-91$ & $58-68$ & $80-90$ & $42-53$ & $1-10$ \\
\hline 3.5 & 3.0 & 2.6 & 3.6 & 4.8 & 3.1 & 3.2 & 4.2 & 5.0 & 3.7 & 3.3 & 4.0 & 3.2 & 3.0 & 2.7 & 4.1 \\
\hline ND & 8.0 & ND & ND & 10 & ND & ND & 10 & ND & ND & ND & 10 & ND & ND & 7.8 & ND \\
\hline 3.3 & 3.9 & 4.1 & 4.0 & 4.2 & 4.2 & 4.1 & 5.1 & 4.9 & 5.0 & 4.8 & 5.0 & 3.3 & 3.2 & 3.3 & 3.5 \\
\hline 1.15 & 1.4 & 1.1 & 1.3 & 1.7 & 1.3 & 1.2 & 1.8 & 1.5 & 1.2 & 1.4 & 1.7 & 1.4 & 1.3 & 1.5 & 1.25 \\
\hline ND & 2.53 & ND & ND & 1.27 & ND & ND & 1.63 & ND & ND & ND & 2.42 & ND & ND & 1.38 & ND \\
\hline 3.7 & 3.4 & 3.0 & 3.6 & 3.2 & 3.4 & 3.1 & 4.0 & 4.4 & 3.9 & 3.8 & 3.4 & 2.7 & 3.1 & 2.9 & 3.8 \\
\hline 0.54 & 0.58 & 0.47 & 0.67 & 0.67 & 0.67 & 0.59 & 0.76 & 0.72 & 0.77 & 0.64 & 0.66 & 0.57 & 0.57 & 0.55 & 0.67 \\
\hline
\end{tabular}

Table 2. (Continued).

\begin{tabular}{|c|c|c|c|c|c|c|c|c|c|c|c|c|c|c|}
\hline \multicolumn{8}{|c|}{483} & \multicolumn{7}{|c|}{$483 \mathrm{~B}$} \\
\hline 17 & 20 & 21 & 22 & 23 & 25 & 26 & 26 & 4 & 7 & 8 & 12 & 13 & 17 & 19 \\
\hline 2 & 1 & 2 & 4 & 2 & 1 & 2 & 3 & 5 & 2 & 3 & 1 & 3 & 1 & 2 \\
\hline $120-128$ & $118-127$ & $43-51$ & $117-127$ & $92-103$ & $48-58$ & $140-149$ & $98-108$ & $13-23$ & $8-19$ & $26-35$ & $40-46$ & $64-74$ & $138-146$ & $21-30$ \\
\hline 2.8 & 6.2 & 5.5 & 3.6 & 6.4 & 4.3 & 6.2 & 5.1 & 2.6 & 5.6 & 1.6 & 3.7 & 6.4 & 5.5 & 5.7 \\
\hline ND & ND & ND & 9.8 & ND & ND & 13 & 16 & ND & ND & ND & ND & 16 & ND & ND \\
\hline 2.3 & 5.2 & 5.5 & 4.8 & 5.0 & 5.8 & 5.6 & 5.7 & 2.2 & 4.0 & 2.1 & 4.1 & 5.2 & 5.5 & 5.7 \\
\hline 0.70 & 2.0 & 2.3 & 1.6 & 1.95 & 1.9 & 2.0 & 2.1 & 1.3 & 1.4 & 1.2 & 1.6 & 2.0 & 2.0 & 2.1 \\
\hline ND & ND & ND & 2.19 & ND & ND & 2.65 & 1.73 & ND & ND & ND & ND & 1.61 & ND & ND \\
\hline 3.5 & 5.2 & 2.6 & 3.0 & 4.3 & 5.0 & 5.3 & 4.7 & 3.6 & 2.6 & 2.4 & 4.4 & 3.8 & 4.4 & 5.5 \\
\hline 0.53 & 0.93 & 1.0 & 0.48 & 0.75 & 1.0 & 0.84 & 0.97 & 0.46 & 0.7 & 0.43 & 0.78 & 0.7 & 1.14 & 1.2 \\
\hline
\end{tabular}

Table 2. (Continued).

\begin{tabular}{|c|c|c|c|c|c|c|c|c|c|c|c|c|c|c|c|}
\hline \multicolumn{16}{|c|}{$485 \mathrm{~A}$} \\
\hline 23 & 23 & 24 & 25 & 23 & 29 & 30 & 30 & 31 & 33 & 34 & 35 & 35 & 38 & 39 & 39 \\
\hline 2 & 3 & 2 & 1 & 1 & 3 & 2 & 1 & 1 & 2 & 2 & 5 & 1 & 2 & 3 & $\stackrel{4}{405-115}$ \\
\hline $38-50$ & $60-72$ & $77-87$ & $63-74$ & $66-76$ & $118-130$ & $116-129$ & $121-134$ & $98-119$ & $7-19$ & $78-89$ & $48-62$ & $21-31$ & $104-119$ & $47-57$ & $105-115$ \\
\hline 5.7 & 4.8 & 4.8 & 4.4 & 4.6 & 4.4 & 3.9 & 5.4 & 4.1 & 4.2 & 3.3 & 4.0 & 3.7 & 3.8 & 4.0 & 3.8 \\
\hline ND & ND & ND & 16 & ND & ND & ND & ND & 12 & ND & ND & ND & ND & 13 & ND & ND \\
\hline 5.6 & 5.4 & 5.2 & 5.7 & 7.0 & 6.1 & 5.1 & 5.7 & 4.0 & 5.2 & 4.4 & 3.9 & 4.1 & 4.1 & 4.7 & 5.4 \\
\hline 2.0 & 2.5 & 2.2 & 2.1 & 2.1 & 1.8 & 1.9 & 2.5 & 1.8 & 2.2 & 1.6 & 1.7 & 1.8 & 1.8 & 1.7 & 1.7 \\
\hline ND & ND & ND & 1.84 & ND & ND & ND & ND & 1.84 & ND & ND & ND & ND & 1.61 & ND & ND \\
\hline 3.9 & 4.1 & 4.2 & 4.9 & 5.4 & 5.6 & 4.6 & 5.7 & 4.2 & 4.6 & 3.2 & 4.1 & 4.6 & 3.0 & 5.6 & 3.7 \\
\hline 0.91 & 1.0 & 0.87 & 1.1 & 1.22 & 0.95 & 0.72 & 1.22 & 0.73 & 1.22 & 0.77 & 0.69 & 0.81 & 0.76 & 1.0 & 0.83 \\
\hline
\end{tabular}




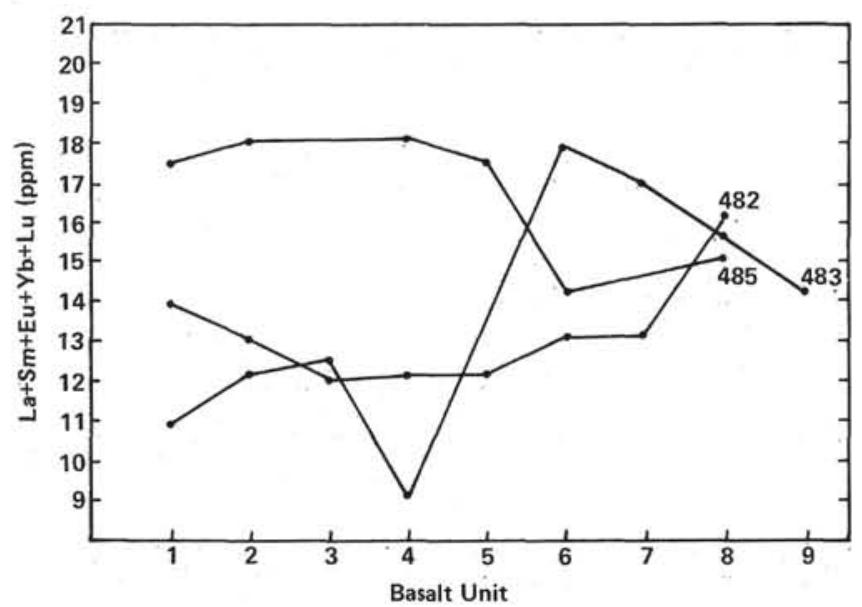

Figure 14. Distribution of the sum of $\mathrm{La}, \mathrm{Sm}, \mathrm{Eu}, \mathrm{Yb}$, and $\mathrm{Lu}$ in basalt units at the various sites. 